\title{
Ellipsoidal Janus Nanoparticles Assembled at Spherical Oil/Water Interfaces
}

\author{
Xuan - Cuong Luu ${ }^{1}$ and Alberto Striolo, ${ }^{2 *}$ \\ ${ }^{1}$ School of Chemical, Biological, and Materials Engineering, University of Oklahoma, Norman, \\ OK 73019, United States \\ ${ }^{2}$ Department of Chemical Engineering, University College London, Torrington Place, \\ London WC1E 7JE, UK
}

\begin{abstract}
The equilibrium behavior of ellipsoidal Janus nanoparticles adsorbed at spherical oil/water interfaces was investigated using dissipative particle dynamics simulations. Several phenomena were documented that were not observed on similar simulations for planar oil/water interfaces. The nanoparticles were found to yield isotropic, radial nematic phases, and axial nematic domains, depending on the nanoparticle characteristics (aspect ratio and surface chemistry), particle density at the interface, and droplet properties (curvature of the interface, and, surprisingly, liquid type). When adsorbed on water droplets, the nanoparticles with high aspect ratio and few nonpolar beads on their surface can show two preferred orientation angles. Only one equilibrium orientation was found for such nanoparticles adsorbed on oil droplets. These observations might help explain a discrepancy previously reported between experimental and simulation results concerning the preferential orientation of particles at liquid-liquid interfaces. Different driving forces are responsible for the phenomena just summarized, including nanoparticle-nanoparticle and nanoparticle-solvent interactions, nanoparticle density at the interface, and droplet curvature via the Laplace pressure. The simulation results we present could be useful for engineering Pickering emulsions towards practical applications, and perhaps also for guiding new technologies for material synthesis.
\end{abstract}

KEYWORDS: Pickering emulsions; Radial nematic phase; Dissipative particle dynamics

* Corresponding author. Email: a.striolo@ucl.ac.uk 


\section{INTRODUCTION}

Particles and nanoparticles (NPs) can be used to stabilize the so called Pickering emulsions. ${ }^{1}$ It has been proposed to use Pickering emulsions in various applications, including biofuel upgrading, ${ }^{2}$ cosmetics and oil processing. ${ }^{3,4}$ Because NPs are used as emulsifiers, their characteristics (e.g., surface chemistry and shape) have critical effects on the emulsions properties (e.g., interfacial tension, droplet size, and emulsion stability). For example, spherical Janus NPs (in which the surface functional groups are segregated in two distinct portions of the NP surface) are more effective at reducing interfacial tension than NPs of similar size and composition in which the functional groups are randomly distributed on the surface (homogeneous NPs). ${ }^{5-7}$ Non-spherical NPs (e.g., rods, sheets, wedges, disk-like, needle-like, etc.) can be more efficient in stabilizing emulsions than spherical NPs, ${ }^{8-10}$ and can also yield oriented structures, which could lead to additional interesting phenomena and possible applications. ${ }^{11-14}$ In our previous work, we focused on NPs with different surface chemistry and shape adsorbed on flat oil/water interfaces. ${ }^{5,6,13}$ We assessed structural and dynamical properties of spherical NPs with different surface chemistry, and also their ability to reduce the interfacial tension. ${ }^{5,6} \mathrm{We}$ simulated ellipsoidal NPs and found that by changing NPs aspect ratio, surface chemistry, and surface density it is possible to obtain either isotropic or axial nematic phases. ${ }^{13}$

In this manuscript we seek to quantify the structure and possible emergent behavior of ellipsoidal Janus NPs when they adsorb on oil (water) droplets immersed in water (oil). By changing the size of the droplets, we quantify how the curvature of the interface affects the NPs behavior, which is expected to have both practical and fundamental interest. ${ }^{15-22}$ Understanding how the curvature affects the orientation of elongated particles at interfaces is crucial when Pickering emulsions are used in catalysis, ${ }^{2}$ has implications for manipulating the stability of particle- 
stabilized emulsion droplets, ${ }^{16,18}$ and could perhaps prove useful for guiding the self-assembly of anisotropic particles into supra-colloidal aggregates. It should be noted that, because of computing power limitations, the droplets considered are never larger than $\sim 10$ times the maximum dimension of the NPs considered.

In the remainder of the manuscript we first present the methods and algorithms; we then report our results, discussing their relevance to experimental and theoretical observations available in the literature; finally, we summarize our main findings.

\section{METHODS and ALGORITHMS}

All simulations were performed implementing the dissipative particle dynamics (DPD) formalism. ${ }^{23}$ The simulation package LAMMPS was used, ${ }^{24}$ and the procedures implemented are fully described in our prior work. ${ }^{5,13}$ Water was modeled as a single bead assumed to contain five water molecules. Oil molecules were modeled by two beads, connected by one harmonic spring. The nanoparticles considered (NPs) are prolate Janus NPs with different aspect ratios and surface chemistry. The equation defining the NP shape is $x^{2} / b^{2}+y^{2} / b^{2}+z^{2} / c^{2}=1$, where $\mathrm{x}, \mathrm{y}$ and $\mathrm{z}$ are Cartesian coordinates, and $b$ and $c$ are the semi-axes of the ellipsoidal NPs. As in our prior works, the NPs are hollow. The surface chemistry is manipulated by placing polar and nonpolar beads on the NP surface. We report the nonpolar fraction $\mathrm{N}$ of the NP surface beads. For example, $\mathrm{N}=30$ indicates that $30 \%$ of the beads on the NP surface are nonpolar.

For the simulations discussed herein we consider one droplet, either oil or water, surrounded by the continuous phase (water or oil). The size of the droplet was varied. At the beginning of each simulation, a number of NPs are randomly placed at the droplet interface with their polar (nonpolar) part in the water (oil) phase. Shown in Figure 1 is an example of one simulated 
system consisting of an oil droplet in water. For each simulation an orthorhombic simulation box with dimensions $L_{X} \times L_{Y} \times L_{Z} \sim 120 \times 120 \times 120 R_{c}^{3}$ was used, where $R_{c}$ is the DPD cutoff distance, and $L_{i}$ is the box length along the $i^{\text {th }}$ direction. Periodic boundary conditions are used in all three dimensions. The largest droplet simulated was of diameter $\sim 85 R_{c}$, which implies that $\sim 35 R_{c}$ was the minimum distance between a droplet and its periodic replica. Given the sort range typical of DPD interactions, we considered the simulation boxes large enough to minimize box-size effects. Each simulation was first equilibrated during $10^{6}$ time steps. During the subsequent $10^{6}$ time steps, data were recorded every 1000 time steps and used for analysis. Each simulation was repeated 3 times with different initial configurations to check the reliability of the results. To ensure that the initial configurations were significantly different from each other, one was prepared with the NPs perpendicular to the interface, and another with the NPs parallel to the interface. The consistency between the simulation results suggests that proper equilibration was achieved.

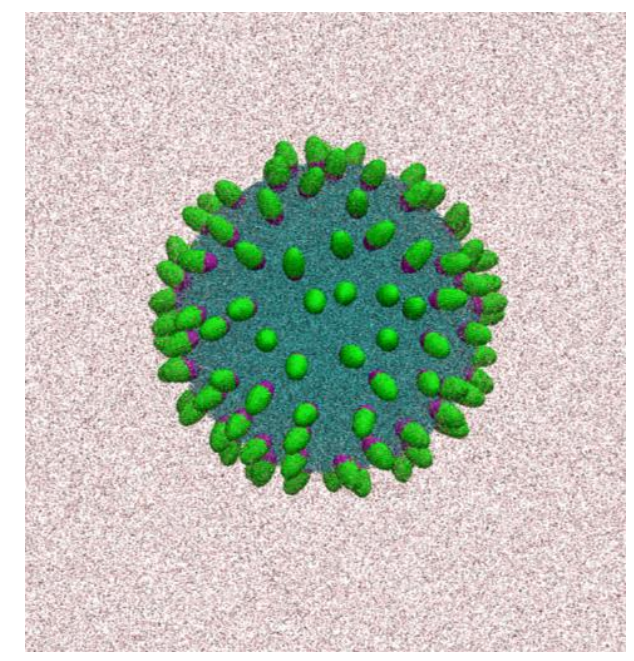

Figure 1. Schematic of one typical simulation box. The oil droplet (cyan) is covered by NPs and immersed in water (pink). Green and purple beads are polar and nonpolar, respectively, on the NPs. The prolate NPs have aspect ratio 2.0 , and $30 \%$ of the beads on their surface are nonpolar. 
To characterize the NPs structure on a droplet we focus on the orientation of their longest axes (the $\mathrm{c}$ axes) either with respect to each other or with respect to the radial directions from the center of the droplet. An isotropic phase is observed when the c axes of the various NPs are randomly oriented. ${ }^{25}$ When the c axes are parallel to each other, an axial nematic phase is observed. ${ }^{13}$ A radial nematic phase is formed when the $\mathrm{c}$ axes are parallel to the radial direction of the droplet. ${ }^{26}$ Order parameters can be used to distinguish the various phases. We discussed the axial nematic order parameter when we considered flat interfaces. ${ }^{13}$ For this work the radial nematic order parameter, $S_{R}$, is more relevant, as we used it to discriminate isotropic and radial nematic phases. The latter order parameter is obtained as: ${ }^{26}$

$$
S_{R}=\left\langle\sum_{i} \frac{1}{2}\left(3\left(u_{i} \bullet R_{i}\right)^{2}-1\right)\right\rangle
$$

In Eq. (1), $u_{i}$ is the unit vector along the NP $\mathrm{c}$ axis and $R_{i}$ is the unit vector representing the radial direction from the center of the droplet. Angular brackets represent ensemble averages. $S_{R} \sim 1$ when a radial nematic phase is observed; $S_{R} \sim 0$ when an isotropic phase with respect to the radial direction is observed. The calculation of the radial nematic order parameter facilitates the definition of the orientation angle $\alpha$, which is the angle between $u_{i}$ and $R_{i}$. In Figure 2 we report a schematic. When $\alpha=0^{\circ}\left(90^{\circ}\right)$ the correspondent $\mathrm{NP}$ is parallel (perpendicular) to the droplet radial direction, and therefore perpendicular (parallel) to the local liquid-liquid interface. Note that the angle $\alpha$ is defined so that it is never larger than $90^{\circ}$. 


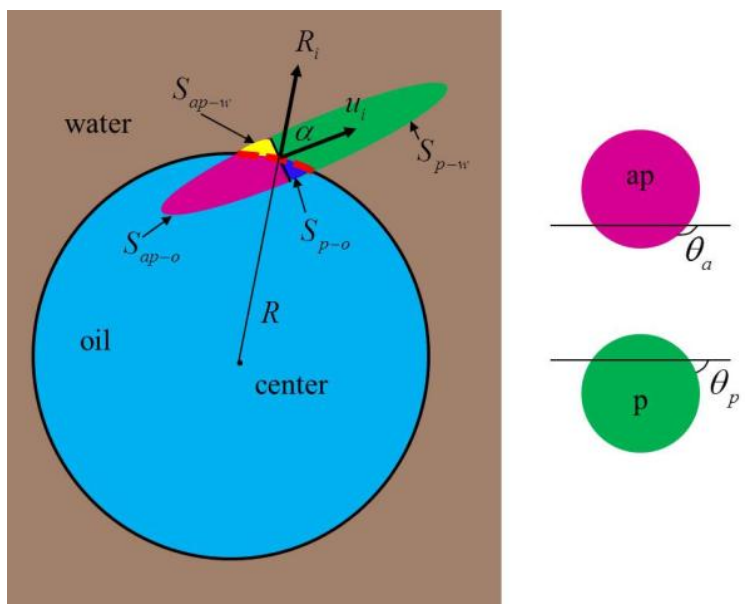

Figure 2. Schematic representing an ellipsoidal Janus NP adsorbed at the oil-water interface. In this picture, ap (pink), $\mathrm{p}$ (green), o (cyan), and w (purple) represent nonpolar, polar, oil, and water, respectively. $u_{i}$ and $R_{i}$ are the unit vectors along the NP c axis and along the radial direction from the center of the droplet, respectively. $S_{p-w}, S_{p-o}, S_{a p-w}$, and $S_{a p-o}$ are polar NP surface in water, polar surface in oil, nonpolar surface in water, and nonpolar surface in oil, respectively. The interfacial area occupied by the NP, $S_{I}$, is highlighted in red. $\theta_{a}$ and $\theta_{p}$ are the contact angles of completely nonpolar and polar spherical NPs, respectively.

\section{RESULTS}

\section{A. Oil Droplets Immersed in Water}

In Table 1 we report $S_{R}$ and the average orientation angle obtained for NPs with aspect ratio $\mathrm{c} / \mathrm{b}=2$ adsorbed on an oil droplet with diameter $\mathrm{d} \sim 50 \mathrm{R}_{\mathrm{c}}$ immersed in water. The NPs have two surface chemistries: $\mathrm{N}=70$ and $\mathrm{N}=30$ ( $70 \%$ and $30 \%$ nonpolar beads, respectively). The radial order parameter was calculated at various NP surface densities, expressed in terms of interface area per NP, $A_{c}$. Low $A_{c}$ corresponds to high surface density. The lowest values of $A_{c}$ for both $\mathrm{N}=30$ and $\mathrm{N}=70 \mathrm{NPs}$ considered here is $\sim 19 R_{c}^{2}$. When more particles are placed at the oil-water interface, a few of the particles desorb during the course of the simulations. When that happened, we deleted the desorbed NPs, as this paper is focused on the NPs adsorbed at the interface. Note that, although in some cases NPs desorbed from liquid-liquid interfaces, we did not investigate 
the adsorption-desorption process. The strong driving forces that drive NPs to liquid-liquid interfaces hinder the latter. The interested reader is referred to Ref. [27] for an experimental investigation on forced NP desorption from liquid-liquid interfaces.

The results in Table 1 show that $S_{R}$ does not change significantly when $\mathrm{A}_{\mathrm{c}}$ varies for either NP type. $S_{R} \sim 0.8$ for NPs with $\mathrm{N}=30$, and $S_{R}<0.4$ for NPs with $\mathrm{N}=70$. These data indicate that NPs with $\mathrm{N}=30$ yield a radial nematic phase, while the $\mathrm{N}=70 \mathrm{NPs}$ yield an isotropic phase. The average orientation angle results show that the $\mathrm{N}=30$ NPs yield an orientation angle $\alpha$ lower than $\sim 20^{\circ}$ with respect to the droplet radial direction. The density of the NPs at the oil-water interface does not affect significantly this orientation. Instead, the average orientation angle for the NPs with $\mathrm{N}=70$ does change with NPs surface density, form $\sim 65^{\circ}$ at low surface density to $\sim 45^{\circ}$ at the highest surface density considered. At all conditions, the N=70 NPs, as already mentioned, yield a radially isotropic phase. The change in $S_{R}$ observed for the $N=70$ NPs in Table 1 is due predominantly to steric effects. These are more pronounced for the $\mathrm{N}=70 \mathrm{NPs}$ than for the $\mathrm{N}=30$ NPs because the latter NPs orient their main axis almost perpendicular to the interface.

The behavior of NPs with aspect ratio 2 on the droplet, just summarized, is similar to that of the same NPs adsorbed at the flat oil/water interface as shown in our previous studies. ${ }^{13}$ The only difference is that instead of radial nematic order vs. isotropic, axial nematic order vs. isotropic was observed at the flat interfaces. 
Table 1. Radial order parameter $S_{R}$, and average orientation angle of NPs adsorbed on an oil droplet of diameter $50 \mathrm{R}_{\mathrm{c}}$ immersed in water. The errors are obtained as one standard deviation from the average of three simulations. NPs have aspect ratio 2 and surface chemistry $N=30$ or 70 .

\begin{tabular}{|c|c|c|c|c|}
\hline \multirow{2}{*}{ Area per NP $\left(\mathrm{A}_{\mathrm{c}}\right)$} & \multicolumn{2}{|c|}{$\mathrm{N}=30$} & \multicolumn{2}{c|}{$\mathrm{N}=70$} \\
\cline { 2 - 5 } & $\mathrm{S}_{\mathrm{R}}$ & $\alpha\left(^{\circ}\right)$ & $\mathrm{S}_{\mathrm{R}}$ & $\alpha\left(^{\circ}\right)$ \\
\hline 90.5 & $0.80 \pm 0.05$ & $19.9 \pm 2.8$ & $0.16 \pm 0.06$ & $65.6 \pm 4.5$ \\
\hline 39.8 & $0.82 \pm 0.03$ & $19.5 \pm 1.5$ & $0.19 \pm 0.04$ & $63.5 \pm 3.8$ \\
\hline 26.5 & $0.82 \pm 0.03$ & $16.8 \pm 1.1$ & $0.15 \pm 0.02$ & $61.7 \pm 2.8$ \\
\hline 19.4 & $0.89 \pm 0.01$ & $10.4 \pm 0.6$ & $0.40 \pm 0.02$ & $45.6 \pm 1.9$ \\
\hline
\end{tabular}

In Figure 3 we report representative simulation snapshots for the systems discussed in Table $\mathbf{1}$ at two surface densities. These images confirm that NPs with $\mathrm{N}=30$ (panels a and b) yield a radial nematic phase and those with $\mathrm{N}=70$ (panels $\mathrm{c}$ and d) yield an isotropic phase, independent on NP surface density. We expect that NPs of the same aspect ratio and with small $\mathrm{N}$ will behave similarly to those NPs with $\mathrm{N}=30$, while NPs with large $\mathrm{N}$ will behave similarly to those NPs with $\mathrm{N}=70$. Some examples are shown in Figure S1 in Supplemental Information (SI) for N=10 NPs and N=90 NPs. It is worth mentioning that the NPs orientation angle at an interface, as well as the NPs structure, both depend on the interactions between the NP and the solvent beads. In Figure S2 in SI, for example, we show that it is possible to change NP structures from isotropic to radial nematic by reducing the repulsive interaction between nonpolar beads on the NP and oil beads. Experimentally, changes in effective interactions can be achieved by either grafting different the functional groups to the NP surface, or by changing the solvents. 

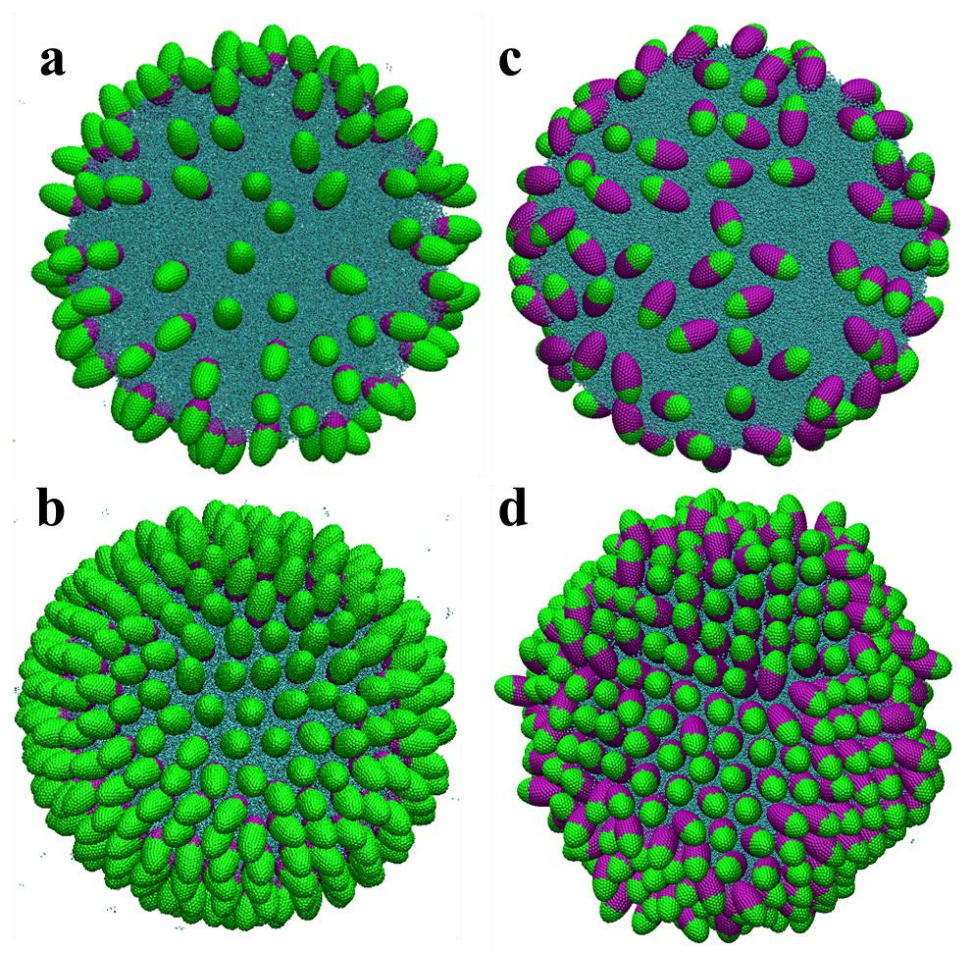

Figure 3. Representative simulation snapshots for ellipsoidal Janus NPs adsorbed on one oil droplet immersed in water. The color code is that of Figure 1. Water beads are not shown for clarity. The droplet diameter is $50 \mathrm{R}_{\mathrm{c}}$. The NPs have aspect ratio 2 . Left and right panels are for

NPs with $\mathrm{N}=30$ and $\mathrm{N}=70$, respectively. Top and bottom panels are for $A_{c}=90.5 R_{c}^{2}$ and

$$
A_{c}=19.4 R_{c}^{2} \text {, respectively. }
$$

To further quantify the NP packing structure on the curved interface, we extracted a portion of the droplet surfaces from panels (b) and (d) in Figure 3. We then magnified these images in panels (a) and (c) in Figure 4, respectively. To improve visualization we flattened these curved surfaces and showed the locations of the NPs center of mass in panels (b) and (d), respectively. The algorithm implemented for flattening the interfaces is described by Meng et al. ${ }^{28}$ To quantify the results we considered the nearest neighbors to one tagged NP. Two NPs are considered nearest neighbors if the distance between their two centers is less than $5.5 R_{c}$, the shortest distance between two NPs parallel to each other. In Figure 4, the black filled circles represent NPs with six nearest neighbors; the stars indicate NPs that do not have six nearest neighbors. 
The results in Figure 4 suggest that both N=30 and N=70 NPs yield hexagonal structures on the oil droplet, although defects are visible. The defects are due, in part, to elastic stress due to the curvature of the interface. This result agrees with observations by Bausch et al., ${ }^{29}$ who investigated spherical particles adsorbed on oil droplets, and found that the defects are necessary to alleviate the elastic stress induced by the adsorption of repulsive particles at the interface. Although we did not quantify this aspect, it is expected that the ordered domains decrease in size as the droplet diameter decreases. The defects can also be a consequence of the ellipsoidal shape of the NPs considered in our simulations, which might prevent hexagonal packing when the particles are not perpendicular to the interface.

a

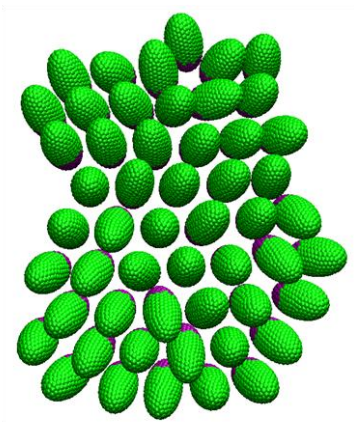

b

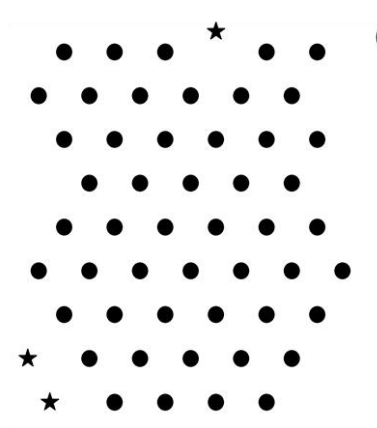

c
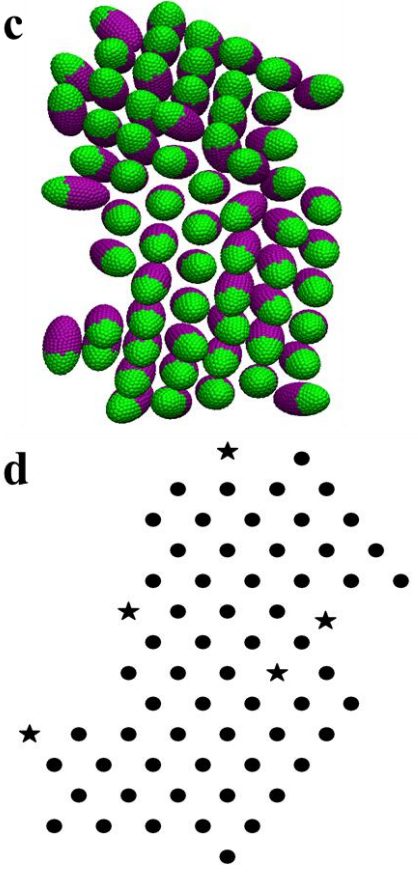

Figure 4. One portion of the droplets surface shown in panels (b) and (d) of Figure 3 is extracted and magnified in panels (a) and (c) of this figure, respectively. The interfacial areas are flattened and shown in panels (b) and (d), respectively. In these latter panels the filled circles are for the centers of NPs that have six nearest neighbors, the stars for NPs with more or less than six nearest neighbors, which are indicative of 'defects'. 
When NPs of aspect ratio 4 adsorb at the flat oil/water interface, their structure changes from isotropic to axial nematic upon increasing their surface density. ${ }^{13}$ We investigate here whether the curved interface affects the behavior of such NPs (with aspect ratio 4). In Figure 5 we report representative simulation snapshots obtained for such NPs adsorbed on an oil droplet of diameter $50 \mathrm{R}_{\mathrm{c}}$. The left (right) panels are for NPs with $\mathrm{N}=30(\mathrm{~N}=70)$. An isotropic phase is observed when NP density is low $\left(\mathrm{A}_{c} \sim 90 R_{c}^{2}\right)$ for both $\mathrm{N}=30 \mathrm{NPs}$ (panel a) and $\mathrm{N}=70$ NPs (panel d), as confirmed by low values of $S_{R}\left(S_{R} \sim 0.01\right.$ for both panels). At medium NP density $\left(\mathrm{A}_{\mathrm{c}} \sim 35 R_{c}^{2}\right.$, panels $b$ and e) we observe that some NPs are aligned in the same direction. These NPs are found grouped into 'axial nematic' domains. As domains were not observed on the flat oil-water interfaces, their appearance here must be due to the curved interface. Visualization of simulation snapshots showed that the droplet interface in these domains was flat. Note that the orientation angle of NPs on one domain can be different compared to that on other domains. When the NP density is sufficiently high $\left(\mathrm{A}_{c} \sim 24 R_{c}^{2}\right)$, a radial nematic phase is observed for N=30 NPs (panel c, for which $S_{R} \sim 0.88$ ), while axial nematic domains are observed for N=70 NPs (panel f). In the discussion section, below, we attempt to rationalize how the NP aspect ratio, the droplet curvature, the NP adsorption energy, and NP-fluid interactions determine the NP behavior. 

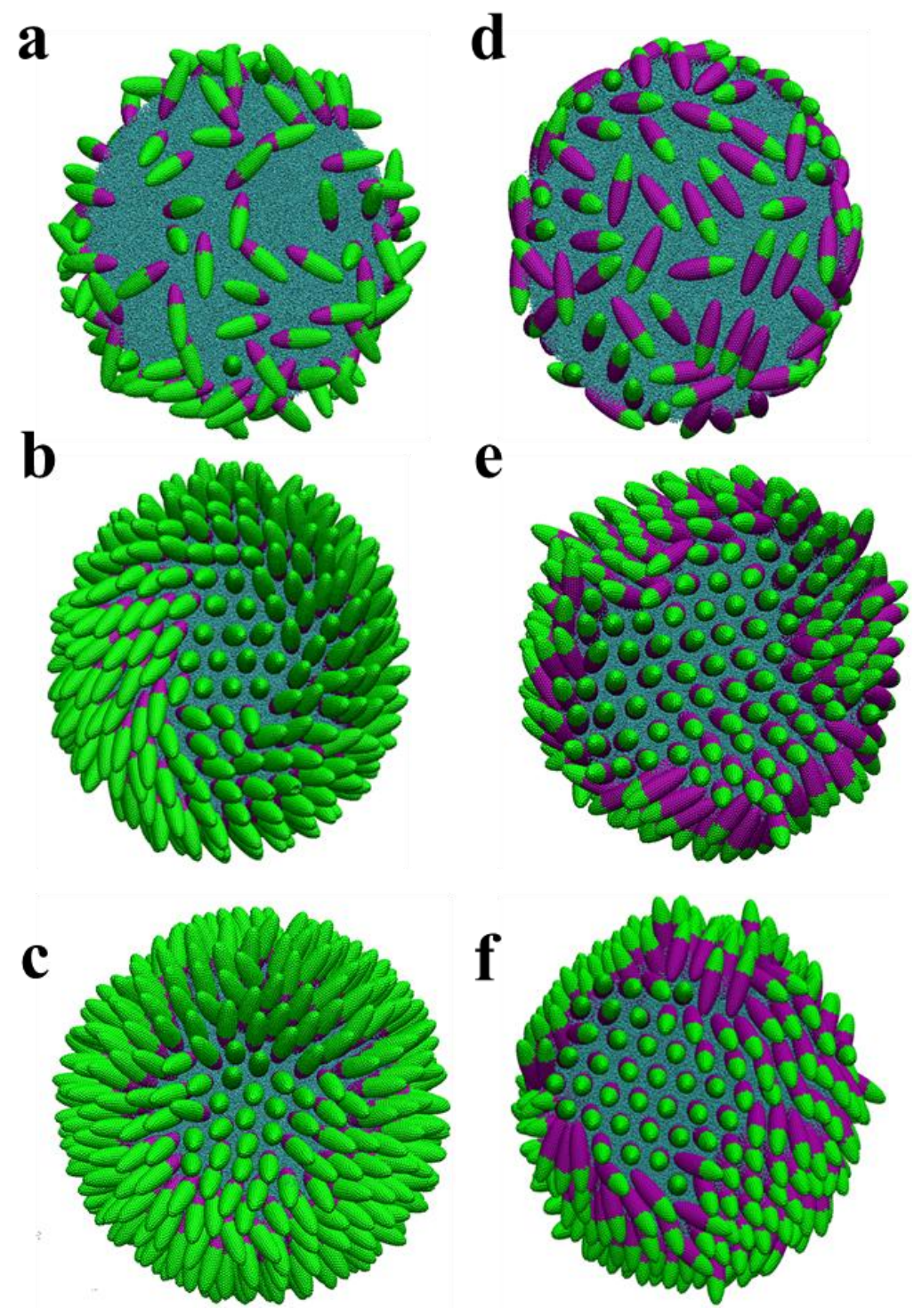

Figure 5. Representative simulation snapshots for ellipsoidal Janus NPs adsorbed on one oil droplet. Water beads are not shown for clarity. The droplet diameter is $50 \mathrm{R}_{\mathrm{c}}$. The NPs have aspect ratio 4. Left and right panels are for NPs with $\mathrm{N}=30$ and $\mathrm{N}=70$, respectively. From top to bottom, the NP surface density is increased: in panels (a) and (d) $\mathrm{A}_{\mathrm{c}} \sim 90 R_{c}^{2}$; (b) and (e)

$$
\mathrm{A}_{\mathrm{c}} \sim 35 R_{c}^{2} \text {; (c) and (f) } \mathrm{A}_{\mathrm{c}} \sim 24 R_{c}^{2} \text {. }
$$

In Figure 6 we report simulations snapshots to systematically visualize the change of NP structures as the oil droplet size varies. All snapshots are shown at high NP surface density $\left(\mathrm{A}_{c} \sim 24 R_{c}^{2}\right)$. NPs with aspect ratio 4 are used. The left and right panels are for NPs with N=30 
and $\mathrm{N}=70$, respectively. From top to bottom, the results are on a flat interface, on a droplet of size $d=75 R_{c}$, and on a droplet of size $d=20 R_{c}$. The inspection of these images suggests that, as the droplet size reduces, $\mathrm{N}=30 \mathrm{NPs}$ structures change from axial nematic on the entire interface (panel a) to axial nematic domains (panel b) to radial nematic phase (panel c). The observations for $\mathrm{N}=70 \mathrm{NPs}$ are similar to those of N=30 NPs except that the transformation to radial nematic phase is not observed.
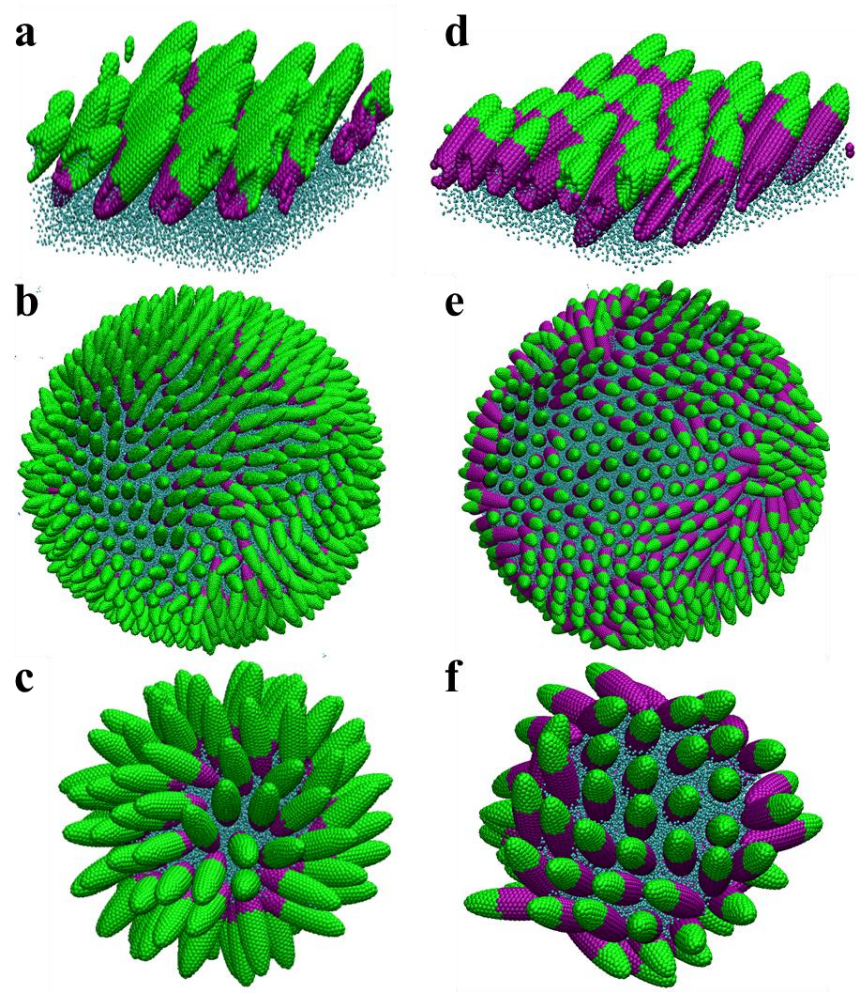

Figure 6. Representative simulation snapshots for ellipsoidal Janus NPs adsorbed at oil/water interfaces of increasing curvature. The NPs have aspect ratio 4 . The area per NP is constant at $\mathrm{A}_{\mathrm{c}} \sim 24 R_{c}^{2}$. Left and right panels are for NPs with $\mathrm{N}=30$ and $\mathrm{N}=70$, respectively. Panels (a) and (d) are for flat interface; (b) and (e) for a droplet of diameter $d=75 R_{c}$; (c) and (f) for a droplet of diameter $\mathrm{d}=20 \mathrm{R}_{\mathrm{c}}$

For the NPs of aspect ratio $\mathrm{c} / \mathrm{b}=4$ and $\mathrm{N}=30$ adsorbed on an oil droplet we systematically changed the droplet size. From an experimental point of view, the droplet size could change 
during evaporation processes, during which the droplet size changes with time. ${ }^{30}$ In Figure 7 we report the average orientation angle, $\alpha$, as a function of the area per NP. The orientation angles at low surface density are similar for all droplet diameters, and they reduce as the area per NP decreases. The effect is more pronounced for smaller droplets. For example, as $A_{c}$ reduces from $\mathrm{A}_{c}=90$ to $20 R_{c}^{2}$, the orientation angles reduce by about $40^{\circ}$ when the NPs are adsorbed on droplets of diameter $\mathrm{d}=20$ and $50 \mathrm{R}_{\mathrm{c}}$, and only by about $20^{\circ}$ when the droplet diameter is $d=75 R_{c}$, or larger (see limit for flat interface). These results suggest that curvature has a considerable effect on the particles behavior when droplet diameter is $50 \mathrm{R}_{\mathrm{C}}$ or less.

We complement our analysis by drawing a 'master curve' (solid line) in Figure 7. This curve identifies three regions corresponding to three NP structures, i.e., isotropic (Region 1), axial nematic domains (Region 2), and radial nematic (Region 3). In each region, the line was obtained by the least square method from the correspondent simulation results. In Region 1 the NP surface density has a negligible effect on the averaged orientation angle. In Region 2 the averaged orientation angle decreases as the NP surface density increases, but the change never exceeds $20^{\circ}$, and it is not uniform for all the particles on a droplet (because of the formation of the axial nematic domains). In Region 3, upon the formation of the radial nematic structure, the averaged orientation angle can change up to $\sim 40^{\circ}$ with a small change in NP surface density.

The results just discussed are most likely dependent on the NP features. In Figure S3 of the Supplemental Information we report simulation results for NPs of aspect ratio 4 with N=70 adsorbed on either a flat oil-water interface, or on one oil droplet of size $d=50 R_{C}$. As the NP surface density increases, the orientation angle changes, by at most $\sim 20^{\circ}$, but it is never less than $40^{\circ}$. This happens because the NPs considered do not yield the radial nematic structure at the conditions considered in our simulations. 


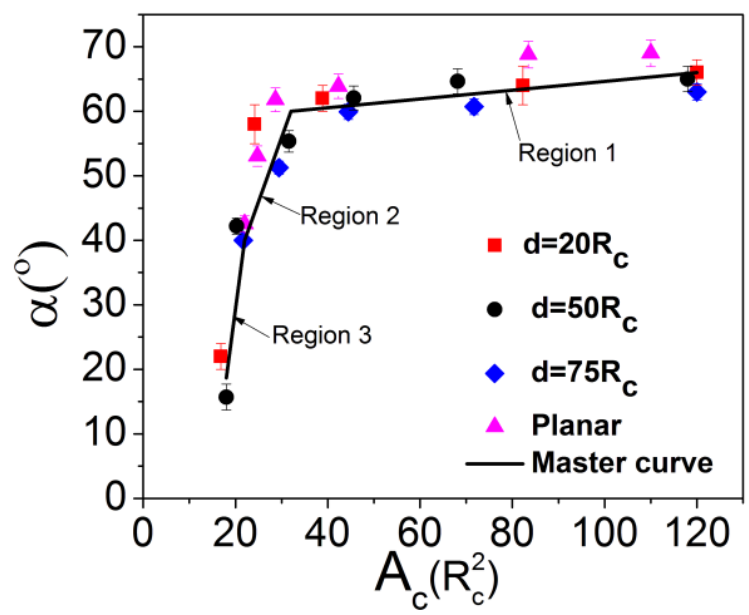

Figure 7. Average orientation angle as a function of area per NP. Results are for NPs with aspect ratio $\mathrm{c} / \mathrm{b}=4$ and $\mathrm{N}=30$. Different symbols are for different droplet diameters. The error bars are obtained as one standard deviation from the average of three simulations. The solid line is a 'master curve', which can be divided into three regions. See text for a discussion.

\section{B. Water Droplets Immersed in Oil}

When the NPs are adsorbed on water droplets immersed in oil the simulation results are qualitatively similar to those discussed above for oil droplets immersed in water. In supplemental material we provide the relevant information (Table S1 and Figures S4-S6). However, there are a few differences. The NPs were found to show only one preferred orientation angle when adsorbed on oil droplets regardless of droplet sizes, NPs type, and surface densities. When NPs of small aspect ratio $(\mathrm{c} / \mathrm{b}=2)$ adsorb on water droplets, they also show only one preferred orientation angle (see Figure S4). However, when NPs of high aspect ratio $(\mathrm{c} / \mathrm{b}=4)$ adsorb on water droplets, they can exhibit two preferred orientation angles (see Figure S5). To qualify this phenomenon we computed the probability distribution function (PDF) of orientation angle $\alpha$ (see Figure 8). We show results for NPs with $\mathrm{c} / \mathrm{b}=4$ and $\mathrm{N}=30$ (panel a) and $\mathrm{N}=70$ (panel $\mathrm{b}$ ). The droplet diameter is $\sim 40 \mathrm{R}_{\mathrm{c}}$. For the NPs with $\mathrm{N}=30$ we observe preferential orientations at $\alpha \sim 75^{\circ}$ and $\alpha \sim 15^{\circ}$, with their prevalence depending on surface density (as the density 
increases the prevalence of the small angle increases). Sequences of visualization snapshots (see, e.g., Figure S5) show that these NPs can dynamically change their preferential orientation during the length of our simulations, suggesting that the results are not due to long-lived metastable states. When the surface density of the NPs is increased (see simulation snapshots in Figure S6) two preferential orientations might be needed to accommodate the structure of the NPs on the droplet surface.

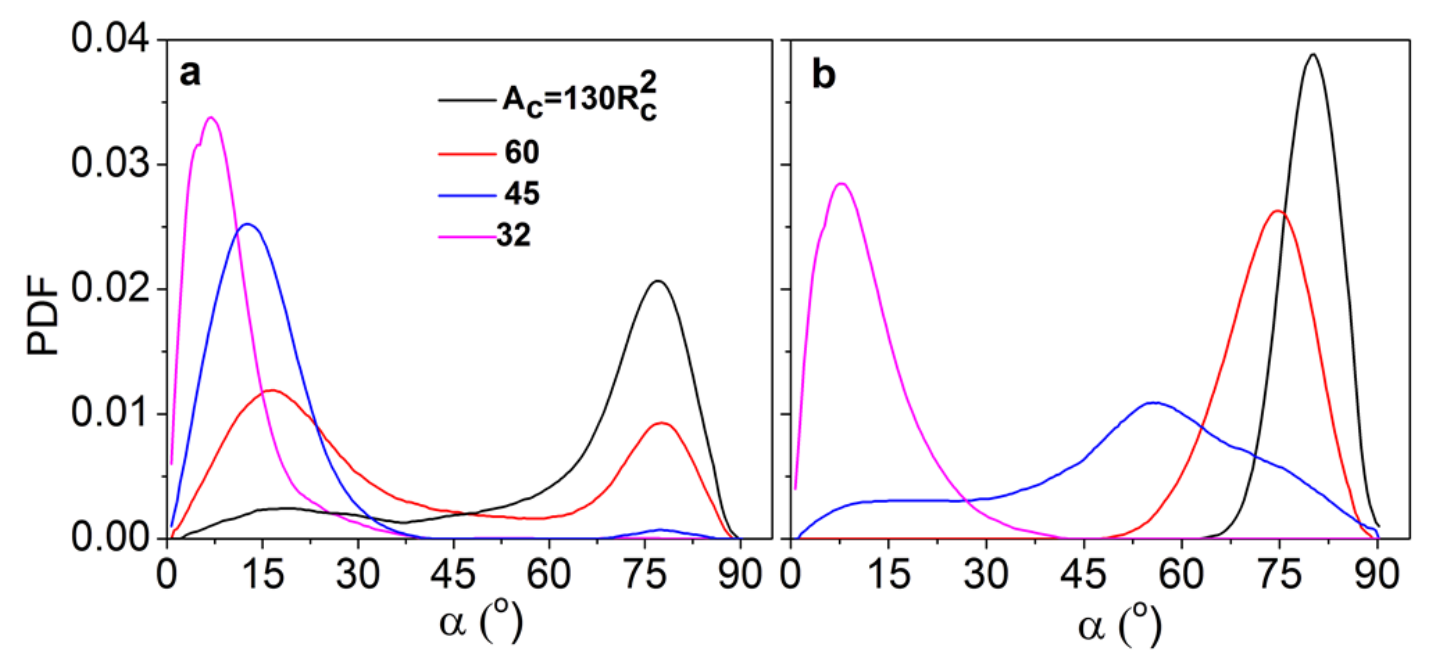

Figure 8. Probability density distribution (PDF) of the orientation angle of ellipsoidal Janus NPs of aspect ratio 4 adsorbed on a water droplet of diameter $d=40 R_{c}$. Panels (a) and (b) are for $N=30$ and $\mathrm{N}=70$, respectively. Different lines are for different NP surface densities, $\mathrm{A}_{\mathrm{c}}$. 


\section{DISCUSSION}

When adsorbed at the flat oil/water interface, NPs arrange to (1) minimize the interfacial energy, (2) minimize particle-particle and particle-solvent interactions, and (3) maximize the system entropy. ${ }^{11,13}$ Similar arguments hold for the attachment of particles to spherical interfaces, with the difference that the interfacial curvature is also responsible for the Laplace pressure, which the particles respond to. The attachment energy for one NP moving from the oil phase to a spherical interface can be derived as (see SI):

$$
\Delta E=-\gamma\left(S_{a p-w} \cos \theta_{a}+S_{p-w} \cos \theta_{p}+S_{I}\right)+\frac{2 \varepsilon \gamma}{R} V_{N P-w}
$$

In Eq. (2), $S_{p-w}, S_{a p-w}$, and $S_{I}$ are the NP polar area immersed in water, NP nonpolar area immersed in water, and droplet interfacial area occupied by the NP, respectively. $\theta_{a}$ and $\theta_{p}$ are the three-phase contact angles of a spherical NP when its surface is totally covered by nonpolar and polar beads, respectively. See Figure $\mathbf{2}$ for a schematic for the various geometrical terms. $\gamma$ is the oil/water interfacial tension. $\varepsilon$ is -1 when for an oil droplet in water, and 1 for a water droplet in oil. $\mathrm{V}_{\mathrm{NP}-\mathrm{w}}$ is the NP volume immersed in water. $\mathrm{R}$ is the droplet radius. The parenthetical terms represent the attachment energy when the interface is flat, while the last term represents the contribution of the Laplace pressure.

The change in NP structure in Figure 5 (isotropic - axial nematic domains - radial nematic) as NP surface density increases is rationalized by contributions from entropic effect, attachment energy reduction, and steric effect.

The entropic effect is responsible for the change from isotropic phase to the axial nematic domain structure. This transition is reminiscent of the one from isotropic to axial nematic we 
documented on flat interfaces, ${ }^{13}$ and for the system considered here it is due to the curvature of the interface.

The transition between axial nematic domains to radial nematic phase that occurs for N=30 NPs (panel (c) in Figure 5) is due to both energetic and steric effects. As all the particles assume low orientation angles, they reduce steric repulsions among themselves, and they also increase the polar NP surface area exposed to water and the apolar one exposed to the oil phase. Note that in the radial nematic phase, most of N=30 NPs are immersed in water, outside of the droplet.

The transformation to radial nematic phase only occurs when the droplet is sufficiently small, and the appropriate NPs are used $(\mathrm{c} / \mathrm{b}=4$ and $\mathrm{N}=30$ for the simulations of Figure 6). This suggests that the radial nematic phase is due to a combination of steric effects and Laplace pressure contributions, both minimized in small droplets when the particles are largely immersed in the surrounding continuous phase.

Park et al., ${ }^{11}$ showed that an ellipsoidal Janus NP adsorbed at the flat oil/water interface can show two preferential orientation angles, correspondent to the energy wells. One energy well is a consequence of NP maximizing the interfacial area occupied, $S_{I}$ (large angle), the other corresponds to the NP minimizing the interactions with the solvents (low angle). A particle can move from one preferential orientation to the other if the energy barrier between the two energy wells is not too high. Our previous results for NPs at a flat oil-water interface suggested that, under the simulated conditions, the NPs only showed one preferential orientation angle. ${ }^{13}$ The results shown in Figure 8 show that when the NPs are adsorbed on a water droplet, they can change their preferential orientation. Our computational analysis (see Figure S8) shows that reducing the size of a droplet induces the presence of two wells in the energy landscape. The energy barrier that separates the two correspondent preferential orientations is of the order of the 
thermal motion, thus explaining why in our simulations it was possible to observe the NPs assuming two preferential orientations only in small water droplets immersed in oil. This observation is probably due to both particle-fluid interactions and to the Laplace pressure.

\section{CONCLUSIONS}

The structure of prolate Janus nanoparticles assembled at spherical oil/water interfaces was investigated using dissipative particle dynamics simulations. The resultant structures were found to depend on nanoparticle characteristics (aspect ratio, surface chemistry, and surface density) and on the properties of the droplets (curvature and liquid type). The NPs can yield isotropic structures, radial nematic phases, or axial nematic domains. A combination of entropic, steric, and energetic effects is responsible for the resultant structures. Our findings can contribute to designing methods for enhancing the stability of Pickering emulsions, using solid particles in phase-transfer catalytic processes, and perhaps designing novel supra-molecular materials obtained via the adsorption of particles on curved interfaces.

\section{ASSOCIATED CONTENT}

\section{Supporting Information}

Attachment energy calculation and relevant radial order parameters, orientation angles and snapshots of NPs adsorbed on the either oil or water droplets at various conditions considered. This material is available free of charge via the Internet at http://pubs.acs.org

\section{ACKNOWLEDGEMENTS}

This work was supported, in part, by NSF award nos. CBET-1033129 and CBET-1247904 to the University of Oklahoma. Generous allocations of computing time were provided by the OU 
Supercomputing Center for Education and Research (OSCER) and by the National Energy

Research Scientific Computing Center (NERSC) at Lawrence Berkley National Laboratory. NERSC is supported by the DOE Office of Science under Contract No. DE-AC02-05CH11231.

\section{REFERENCES}

(1) Binks, B. P. Particles as surfactants—similarities and differences. Curr. Opin. Colloid Interface Sci. 2002, 7, 21-41.

(2) Crossley, S.; Faria, J.; Shen, M.; Resasco, D. E. Solid Nanoparticles that Catalyze Biofuel Upgrade Reactions at the Water/Oil Interface. Science 2010, 327, 68-72.

(3) Sessa, M.; Balestrieri, M. L.; Ferrari, G.; Servillo, L.; Castaldo, D.; D'Onofrio, N.; Donsi, F.; Tsao, R. Bioavailability of encapsulated resveratrol into nanoemulsion-based delivery systems. Food Chem. 2014, 147, 42-50.

(4) Son, H.; Kim, H.; Lee, G.; Kim, J.; Sung, W. Enhanced oil recovery using nanoparticlestabilized oil/water emulsions. Korean J. Chem. Eng. 2014, 31, 338-342.

(5) Luu, X.-C.; Yu, J.; Striolo, A. Nanoparticles Adsorbed at the Water/Oil Interface: Coverage and Composition Effects on Structure and Diffusion. Langmuir 2013, 29, 7221-7228.

(6) Fan, H.; Striolo, A. Nanoparticle effects on the water-oil interfacial tension. Phys. Rev. E. 2012, 86, 051610-051621.

(7) Binks, B. P.; Fletcher, P. D. I. Particles Adsorbed at the Oil-Water Interface: A Theoretical Comparison between Spheres of Uniform Wettability and "Janus" Particles. Langmuir 2001, $17,4708-4710$.

(8) Madivala, B.; Vandebril, S.; Fransaer, J.; Vermant, J. Exploiting particle shape in solid stabilized emulsions. Soft Matter 2009, 5, 1717-1727. 
(9) Mejia, A. F.; Diaz, A.; Pullela, S.; Chang, Y.-W.; Simonetty, M.; Carpenter, C.; Batteas, J. D.; Mannan, M. S.; Clearfield, A.; Cheng, Z. Pickering emulsions stabilized by amphiphilic nanosheets. Soft Matter 2012, 8, 10245-10253.

(10) Dendukuri, D.; Hatton, T. A.; Doyle, P. S. Synthesis and Self-Assembly of Amphiphilic Polymeric Microparticles. Langmuir 2006, 23, 4669-4674.

(11) Park, B. J.; Lee, D. Configuration of nonspherical amphiphilic particles at a fluid-fluid interface. Soft Matter 2012, 8, 7690-7698.

(12) Tu, F.; Park, B. J.; Lee, D. Thermodynamically Stable Emulsions Using Janus Dumbbells as Colloid Surfactants. Langmuir 2013, 29, 12679-12687.

(13) Luu, X.-C.; Yu, J.; Striolo, A. Ellipsoidal Janus Nanoparticles Adsorbed at the Water-Oil Interface: Some Evidence of Emergent Behavior. J. Phys. Chem. B 2013, 117, 13922-13929.

(14) Kalashnikova, I.; Bizot, H.; Bertoncini, P.; Cathala, B.; Capron, I. Cellulosic nanorods of various aspect ratios for oil in water Pickering emulsions. Soft Matter 2013, 9, 952-959.

(15) Cheng, T.-L.; Wang, Y. U. Shape-anisotropic particles at curved fluid interfaces and role of Laplace pressure: A computational study. J. Colloid Interface Sci. 2013, 402, 267-278.

(16) Cui, M.; Emrick, T.; Russell, T. P. Stabilizing Liquid Drops in Nonequilibrium Shapes by the Interfacial Jamming of Nanoparticles. Science 2013, 342, 460-463.

(17) Fan, H.; Striolo, A. Mechanistic study of droplets coalescence in Pickering emulsions. Soft Matter 2012, 8, 9533-9538.

(18) Guzowski, J.; Tasinkevych, M.; Dietrich, S. Capillary interactions in Pickering emulsions. Phys. Rev. E. 2011, 84, 031401-031412.

(19) Hirose, Y.; Komura, S.; Nonomura, Y. Adsorption of Janus particles to curved interfaces. J. Chem. Phys. 2007, 127, 054707-054713.

(20) Kralchevsky, P. A.; Ivanov, I. B.; Ananthapadmanabhan, K. P.; Lips, A. On the Thermodynamics of Particle-Stabilized Emulsions: Curvature Effects and Catastrophic Phase Inversion. Langmuir 2004, 21, 50-63. 
(21) Leandri, J.; Wurger, A. Trapping energy of a spherical particle on a curved liquid interface. J. Colloid Interface Sci. 2013, 405, 249-255.

(22) Lewandowski, E. P.; Bernate, J. A.; Searson, P. C.; Stebe, K. J. Rotation and Alignment of Anisotropic Particles on Nonplanar Interfaces. Langmuir 2008, 24, 9302-9307.

(23) Groot, R. D.; Warren, P. B. Dissipative particle dynamics: Bridging the gap between atomistic and mesoscopic simulation. J. Chem. Phys. 1997, 107, 4423-4435.

(24) Plimpton, S. Fast Parallel Algorithms for Short-Range Molecular Dynamics. J. Comput. Phys. 1995, 117, 1-19.

(25) Fan, C.-p.; Stephen, M. J. Isotropic-Nematic Phase Transition in Liquid Crystals. Phys. Rev. Lett. 1970, 25, 500-503.

(26) Jayasri, D.; Sairam, T.; Murthy, K. P. N.; Sastry, V. S. S. Liquid crystal films on curved surfaces: An entropic sampling study. Phys Stat Mech Appl 2011, 390, 4549-4554.

(27) Garbin, V.; Crocker, J. C.; Stebe, K. J. Forced Desorption of Nanoparticles from an OilWater Interface. Langmuir 2012, 28, 1663-1667.

(28) Meng, G.; Paulose, J.; Nelson, D. R.; Manoharan, V. N. Elastic Instability of a Crystal Growing on a Curved Surface. Science 2014, 343, 634-637.

(29) Bausch, A. R.; Bowick, M. J.; Cacciuto, A.; Dinsmore, A. D.; Hsu, M. F.; Nelson, D. R.; Nikolaides, M. G.; Travesset, A.; Weitz, D. A. Grain Boundary Scars and Spherical Crystallography. Science 2003, 299, 1716-1718.

(30) Bigioni, T. P.; Lin, X.-M.; Nguyen, T. T.; Corwin, E. I.; Witten, T. A.; Jaeger, H. M. Kinetically driven self assembly of highly ordered nanoparticle monolayers. Nat Mater 2006, 5, 265-270. 